\title{
Observations of Chemical Abundances in Stars: Globular Cluster Stars
}

\author{
Robert P. Kraft \\ UCO/Lick Observatory, University of California, Santa Cruz, CA \\ 95064, U.S.A.
}

\begin{abstract}
The chemical composition of globular cluster stars is briefly reviewed, attention being given principally to the following problems: (1) primordial and evolutionary abundance variations among globular cluster stars, and (2) abundance differences between cluster and halo field giants.
\end{abstract}

\section{Primordial and evolutionary abundance variations}

The subject of abundance variations among globular cluster (GC) stars has a long history running back nearly 30 years, and only a brief summary can be given here. More nearly complete treatments can be found in reviews by e.g., Suntzeff (1993), Kraft (1994), Sneden et al. (1997) and Wallerstein et al. (1997). In this investigator's opinion, the most important results and their interpretation are:

(1) Most clusters exhibit star-to-star variations that reflect the results of proton-capture synthesis: e.g., conversion of ${ }^{12} \mathrm{C}$ into ${ }^{13} \mathrm{C}, \mathrm{C}$ into $\mathrm{N}, \mathrm{O}$ into $\mathrm{N}$, $\mathrm{Ne}$ into $\mathrm{Na}$, and $\mathrm{Mg}$ into $\mathrm{Al}$. The site of such synthesis remains problematical and gives rise to two competing models. In the primordial picture, the synthesis occurred in a previous generation of now-dead cluster stars which "polluted" some of the primordial gas, thus providing a reservoir from which was formed a significant fraction of the low-mass stars we presently observe. In the competing "evolutionary" picture, all presently observed low-mass main sequence (MS) stars began with the same chemical composition. But in this case, the composition is modified as the stellar envelope is mixed into regions near or within the H-burning shell. The extent of the modifications increases with evolutionary state on the red-giant branch (RGB) and may be driven selectively by stellar angular momentum. At least one critical test of these scenarios is hampered by the fact that cluster MS stars are faint: even with the largest telescopes, the required high spectral resolution can be obtained only with the greatest difficulty. Thus, most (but not all! see below), of our knowledge of these variations has been confined largely to GC stars brighter than the horizontal branch (HB).

(2) Principal arguments in favor of the primordial variation scenario are these: Many clusters exhibit a CN-band strength dichotomy down to luminosities well below the HB (e.g., Smith \& Norris 1982) and even onto the MS in the case of 47 Tuc where also is found a correlation between $\mathrm{Na} \mathrm{D}$ and $\mathrm{CN}$ band strengths (Briley et al. 1996). This suggests that $\mathrm{Na}$ is already tracking $\mathrm{N}$ among unevolved stars. In other clusters, e.g, M 5 (Smith et al. 1997), some giants have both large $\mathrm{O}$ and large $\mathrm{N}$ abundances, and in M 92 (Carbon et al. 1982), a few giants have overabundances of $\mathrm{C}$ and $\mathrm{N}$, yet others have abnormally low abun- 
dances of $\mathrm{C}$ and $\mathrm{N}$, all contrary to the average "anticorrelated" behavior of these elements. Among bright giants in numerous GCs, especially those having $[\mathrm{Fe} / \mathrm{H}]$ $<-1$, there is commonly a wide scatter in $\mathrm{O}$ and $\mathrm{Na}$ abundances, but these elements are anticorrelated, as one would expect if proton captures convert $\mathrm{O}$ into $\mathrm{N}$ in the same sites where $\mathrm{Ne}$ is transformed into Na (e.g., Kraft et al. 1997, Langer et al. 1997, Denissenkov \& Weiss 1996). Yet the Na scatter exists to the same degree (and presumably anticorrelated $O$ persists with it) among giants well down the giant branch (Pilachowski et al. 1996, Sneden et al. 2000, Cavallo $\&$ Nagar 2000) to a point below the place where deep mixing due to meridional circulation is permitted (Sweigart \& Mengel 1979). A similar anticorrelation of $\mathrm{Mg}$ and $\mathrm{Al}$ exists among some of the bright giants in M 15 and M 13 especially (Shetrone 1996a) (together with a corresponding correlation of $\mathrm{Al}$ with $\mathrm{Na}$ ), and it appears that the enhancement of $\mathrm{Al}$ occurs at the expense of ${ }^{24} \mathrm{Mg}$ (Shetrone $1996 \mathrm{~b}$ ). This is hard to understand if deep mixing is responsible: even among the most metal-poor giants, hydrogen shell temperatures do not exceed $T_{9} \sim 55$, yet synthesis of ${ }^{27} \mathrm{Al}$ from ${ }^{24} \mathrm{Mg}$ requires $T_{9} \sim 70$ (Langer et al. 1997, Cavallo et al. 1998). This conclusion supports the idea that proton captures via the chain that carries ${ }^{24} \mathrm{Mg}$ to ${ }^{27} \mathrm{Al}$ must have taken place within stars more massive than those presently evolving in GCs, and the correlation between $\mathrm{Al}$ and Na suggests that synthesis of $\mathrm{Na}$ must also have taken place in that environment. Finally, specific attention must be called to $r$-process species that could not have been altered within the presently observed cluster giants. In $\mathrm{M} 15([\mathrm{Fe} / \mathrm{H}] \sim-2.3)$, one finds that among the bright giants, $[\mathrm{Eu} / \mathrm{Fe}]$-ratios spread over nearly an order of magnitude (Sneden et al. 1997), and the abundance distribution of heavy elements from $\mathrm{Ba}$ to $\mathrm{Dy}$ mimics that of the pure $r$-process (including $\mathrm{Ba}$ and La) (Sneden et al. 2000). The $r$-process abundances, however, bear no relation to the abundances of the light alpha-elements, those thought to be produced principally by Type II supernovae, e.g., $\mathrm{O}$ and $\mathrm{Mg}$.

(3) There is also evidence that some of the abundance variations depend on evolutionary state, and thus take place within the stars we presently observe, presumably as a result of mixing of the envelope into regions near or within the hydrogen-burning shell. Thus one finds a continuous average decline of the [C/Fe]-ratio as one ascends the giant branches of M92 (Langer et al. 1986), NGC 6397 (Briley et al. 1990), M 15 (Trefzger et al. 1983), M 4 and NGC 6752 (Suntzeff 1993). In all clusters studied, the bright giants exhibit ${ }^{12} \mathrm{C} /{ }^{13} \mathrm{C}$-ratios in the range 4-6 (e.g., Ivans et al. 1999). These results are expected even if mixing proceeds only to depths corresponding to the precursor region just ahead of the hydrogen-burning shell (Sweigart \& Mengel 1979). Evidence for still deeper mixing is found especially in the case of M13 (Pilachowski et al. 1996, Hanson et al. 1998). In this cluster one finds that the lowest $\mathrm{Mg}$ and highest $\mathrm{Na}$ and $\mathrm{Al}$ abundances are found among those giants having $\log g<1$, in comparison with those having $\log g>1$, i.e., the degree of depletion of $\mathrm{Mg}$ and enhancement of $\mathrm{Na}$ and $\mathrm{Al}$ depends directly on evolutionary state. In addition, the bright giants showing the most depletion of $\mathrm{Mg}$ and enhancement of $\mathrm{Na}$ and $\mathrm{Al}$ are also the ones described as "super-oxygen-poor", i.e., $[\mathrm{O} / \mathrm{Fe}]<-0.4$, although a small minority shows little evidence of $\mathrm{Mg}$ and $\mathrm{O}$ depletion (Shetrone 1996a). This, is turn, suggests that the degree of deep mixing at any given evolutionary state is "fine-tuned" by some random stellar variable, e.g., angular momentum (Pinsonneault 1997) coupled with turbulent diffusion (Denissenkov \& Tout 2000). 
If deep mixing driven by rotation indeed modifies $\mathrm{C}, \mathrm{N}, \mathrm{O}, \mathrm{Na}, \mathrm{Mg}$ and $\mathrm{Al}$ abundances among bright giants in M 13 (and possibly in M 15 and $\omega$ Cen), it may also drive other evolutionary effects. An increase in the $\mathrm{He} / \mathrm{H}$-ratio of redgiant envelopes would be expected, and the lifetimes of red giants prior to the $\mathrm{He}$ core flash extended. This could affect the luminosity function (as observed: see Langer et al. 2000) and lead to increased mass loss. This in turn would drive the HB descendants of such giants to the blue (one explanation of the so-called "2nd parameter effect"?), as discussed by Sweigart (1997a,b). Some support for these ideas is found when one compares abundances among three clusters of similar metallicity ( $[\mathrm{Fe} / \mathrm{H}] \sim-1.5)$, but differing $\mathrm{HB}$ morphologies: M 3 ("normal" $\mathrm{HB}$ ), M 13 (blue HB) and NGC 7006 (red HB). If the modest depletions of O and $\mathrm{Mg}$ and enhancements of $\mathrm{Na}$ and $\mathrm{Al}$ in $\mathrm{M} 3$ are taken as standard, then M 13 giants exhibit "severe" modifications, whereas giants of NGC 7006 exhibit little, if any, changes (Kraft et al. 1998). Interesting in this connection is the finding (Peterson et al. 1995, and references therein) that rotational velocities of HB stars in M 13 are generally higher than those of M 3; nothing is known of rotational velocities of $\mathrm{HB}$ stars in NGC 7006.

On the other hand, in one other pair of "2nd parameter" clusters, NGC 288 and NGC 362, Shetrone \& Keane (2000) found that, although Na-O and Al-O anticorrelations are common among bright giants in both clusters, the extent of effects that could be attributed to deep mixing are about the same. Enhancements of $\mathrm{Al}$ are compatible with shallow mixing that converts ${ }^{25,26} \mathrm{Mg}$ to ${ }^{27} \mathrm{Al}$, rather than ${ }^{24} \mathrm{Mg}$, a result similar to that suggested in the case of M4 (Ivans et al. 1999). For these clusters, at least, mixing effects among the giants appear to have little influence on HB morphology and thus the "2nd parameter" effect here could well be driven by a difference in cluster ages. Also, Weiss et al. (2000), have noted that models for deeply mixed giants with severely depleted $\mathrm{O}$ are predicted to enhance $\mathrm{Na}$ by amounts larger than are observed, and that this in turn limits the depletion of $\mathrm{H}$ and enhancement of $\mathrm{He}$ in the envelope. This should seriously limit the blueward displacement of HB descendants, though this restriction may be relieved when the effects of non-LTE calculations in Na are taken into account (Gratton et al. 2000).

(4) One concludes that most clusters exhibit primordial variations which, in some cases, undergo modification by deep mixing as evolution proceeds into the giant branch domain. At least two problems remain naggingly in the background. In $\mathrm{M} 13$ (and perhaps $\mathrm{M} 15$ and $\omega \mathrm{Cen}$ ), ${ }^{27} \mathrm{Al}$ is produced at the expense of ${ }^{24} \mathrm{Mg}$ when at least some stars reach luminosities near the red giant tip. But the hydrogen shell temperature is too low to sustain the required proton-capture synthesis. Is it possible that the shell is unstable, and undergoes short episodes of elevated temperature (e.g., Von Rudloff et al. 1988)? Such moments might be required to explain the production of fresh $\mathrm{Li}$, as recently observed in a few bright GC giants (Kraft et al. 1999, Kraft \& Shetrone 2000). But there are concerns within the framework of the primordial picture as well. To call on "pollution" of the pre-existing medium as the driving mechanism is not enough: the special polluted gaseous regions also must have serious deficiences of elements like $\mathrm{O}, \mathrm{Ne}$ and $\mathrm{Mg}$. How are such regions produced and how do they succeed in maintaining their special chemical identity over the timescale required for low mass star formation to take place? 


\section{Abundances in halo field versus globular cluster giants}

It has generally been assumed that halo field stars are chemical surrogates of globular cluster stars at equivalent metallicity. High resolution studies have shown that this is not the case for many elements. Thus halo field giants lying within the metallicity interval $-2.4<[\mathrm{Fe} / \mathrm{H}]<-1.0$ exhibit progressive declines of the ${ }^{12} \mathrm{C} /{ }^{13} \mathrm{C}$-ratio and $[\mathrm{C} / \mathrm{Fe}]$, along with an increase in $[\mathrm{N} / \mathrm{Fe}]$ as evolution proceeds from the main sequence to the tip of the giant branch, similar to that found in M 92, M 15 and NGC 6397, but $\mathrm{O}$ and Na remain unchanged, i.e., there are no $\mathrm{O}$ depletions and $\mathrm{Na}$ enhancements like those found among most $\mathrm{GC}$ giants (Gratton et al. 2000, Kraft et al. 1993, Sneden et al. 1997). Halo field giants in this metallicity interval show correlated behavior between $\mathrm{Mg}$ and $\mathrm{Na}$ (Hanson et al. 1998, Kraft 1999) and subdwarfs of the same metallicity range follow the identically same correlation (Fulbright 2000). This suggests that $\mathrm{Mg}$ and $\mathrm{Na}$ are formed in the same primordial nucleosynthetic sites (Arnett 1971) and further that proton captures on Ne play no role either in the primordial synthesis or in modifications of $\mathrm{Ne}$ and $\mathrm{Na}$ during stellar evolution. Yet cluster giants show generally anticorrelated behavior of $\mathrm{Na}$ and $\mathrm{Mg}$, with $\mathrm{Na}$ abundances that reach values $\sim 0.5$ dex higher than are found among halo field giants. In the same metallicity interval, halo field giants show no evidence for $[\mathrm{Al} / \mathrm{Fe}]-$ ratios that exceed $\sim+0.2$ dex -indeed most stars have $[\mathrm{Al} / \mathrm{Fe}]<0.0$ (e.g., Gratton \& Sneden 1988 ), yet many $\mathrm{GCs}$ contain giants having $[\mathrm{Al} / \mathrm{Fe}]$-ratios exceeding +0.5 , and indeed in some instances ratios that exceed +1.0 (Brown \& Wallerstein 1992, Shetrone 1996a, Sneden et al. 1997, Ivans et al. 1999). A comprehensive explanation for these striking differences between halo field and globular cluster giants has not been forthcoming. Based on the primordial hypothesis, one could imagine that halo field stars were born in now-disrupted clusters that ab initio were less concentrated than the present-day clusters. In that case, the ejecta of high-mass "polluting" stars would have less opportunity to "infect" the regions giving rise to the formation of low-mass stars. In the evolutionary-modification picture, one might imagine that cluster stars have had more opportunity for close encounters than their field star counterparts. If angular momentum drives the deep mixing, and if close encounters succeed in spinning up the participants, then cluster stars might experience more deep mixing than field stars. But if primordial stellar density is the driving factor required in each of these hypotheses, it is puzzling that widespread abundance variations are found in old, low density dwarf spheroidals such as Draco (Shetrone et al. 1998).

\section{References}

Arnett, D. 1971, ApJ, 166, 153

Briley, M. M., et al. 1990, ApJ, 359, 307

Briley, M. M., et al. 1996, Nature, 383, 604

Brown, J. A., \& Wallerstein, G. 1992, AJ, 104, 1818

Carbon, D., et al. 1982, ApJS, 49, 207

Cavallo, R. M., Sweigart, A. V., \& Bell, R. A. 1998, ApJ, 492, 575

Cavallo, R. M., \& Nagar, N. M. 2000, ApJ (in press) 
Denissenkov, P. A., \& Weiss, A. 1996, A\&A, 308, 773

Dennisenkov, P. A., \& Tout, C. A. 2000, MNRAS, in press

Fulbright, J. 2000, AJ, 120, in press

Gratton, R. G., \& Sneden, C. 1988, A\&A, 204, 193

Gratton, R. G., et al. 2000, A\&A, 354, 169

Hanson, R., et al. 1998, AJ, 116, 1286

Ivans, I., et al. 1999, AJ, 118, 1273

Kraft, R. P. et al. 1993, AJ, 106, 1490

Kraft, R. P. 1994, PASP, 106, 553

Kraft, R. P., et al. 1997, AJ, 113, 279

Kraft, R. P., et al. 1998, AJ, 115, 1500

Kraft, R. P. 1999, Astroph. \& Space Sci., 265, 153

Kraft, R. P. et al 1999, ApJ, 518, L53.

Kraft, R. P., \& Shetrone, M. D. 2000, in Proc. 35th Liege Inter. Astroph. Coll, eds. A. Noels et al. (Liege: Univ. de Liege), p. 177

Langer, G. E., et al. 1986, PASP, 98, 473

Langer, G. E., Hoffman, R. E., \& Zaidins, C. S. 1997, PASP, 109, 244

Langer, G. E., Bolte, M., \& Sandquist, E. 2000, AJ, in press

Peterson, R., Rood, R. T., \& Crocker, D. A. 1995, ApJ, 453, 214

Pilachowski, C., et al. 1996, AJ, 112, 545

Pinsonneault, M. 1997, ARA\&A, 35, 557

Shetrone, M. D. 1996a, AJ, 112, 1517

Shetrone, M. D. 1996b, AJ, 112, 2639

Shetrone, M. D., Bolte, M., \& Stetson, P. B. 1998, AJ, 115, 1888

Shetrone, M. D., \& Keane, M. J. 2000, AJ, 119, 840

Smith, G. H. \& Norris, J. E. 1982, ApJ, 254, 149

Smith, G. H., et al. 1997, PASP, 109, 236

Sneden, C., et al. 1997, AJ, 114, 1964

Sneden, C., Pilachowski, C., \& Kraft, R. P. 2000, AJ, 120, in press

Sneden, C., et al. 2000, ApJ, 536, L85

Suntzeff, N. B. 1993, in ASP Conf. Ser. 48, The Globular Cluster-Galaxy Connection, eds. G. H. Smith \& J. Brodie (San Francisco: ASP), p. 167

Sweigart, A. V. 1997a, ApJ, 474, L23

Sweigart, A. V. 1997b, in The Third Conf. on Faint Blue Stars, IAU Colloq. No. 95, eds. A. G. D. Philip, J. Liebert, R. Saffer \& D. S. Hayes (Schenectady: L. Davis Press), p. 1

Sweigart, A. V., \& Mengel, J. G. 1979, ApJ, 229, 624

Trefzger, C. F., et al. 1983, ApJ, 266, 144

Von Rudloff, I. R., VandenBerg, D. A., \& Hartwick, F. D. A. 1988, ApJ, 324, 840

Wallerstein, G., et al. 1997, Rev. Mod. Phys., 69, 995

Weiss, A. et al. 2000, A\&A, 356, 181 